\title{
Year of Death
}

National Cancer Institute

\section{Source}

National Cancer Institute. Year of Death. NCI Thesaurus. Code C156426.

The year in which an individual died. 